\title{
Potential of fecal microbiota for detection and postoperative surveillance of colorectal cancer
}

Qiulin Yao ${ }^{1 \dagger}$, Meifang Tang ${ }^{1,2+}$, Liuhong Zeng ${ }^{1}$, Zhonghua Chu ${ }^{3}$, Hui Sheng ${ }^{4}$, Yuyu Zhang ${ }^{5}$, Yuan Zhou', Hongyun Zhang ${ }^{1}$, Huayan Jiang ${ }^{5}$ and Mingzhi Ye $e^{1,5,6^{*}}$

\begin{abstract}
Background: Colorectal cancer (CRC) is one of the most common cancers. In recent studies, the gut microbiota has been reported to be potentially involved in aggravating or favoring CRC development. However, little is known about the microbiota composition in CRC patients after treatment. In this study, we explored the fecal microbiota composition to obtain a periscopic view of gut microbial communities. We analyzed microbial 16S rRNA genes from 107 fecal samples of Chinese individuals from three groups, including 33 normal controls (NC), 38 CRC patients (Fa), and $36 \mathrm{CRC}$ post-surgery patients (Fb).

Results: Species richness and diversity were decreased in the Fa and Fb groups compared with that of the NC group. Partial least squares discrimination analysis showed clustering of samples according to disease with an obvious separation between the Fa and NC, and Fb and NC groups, as well as a partial separation between the Fa and $\mathrm{Fb}$ groups. Based on linear discriminant analysis effect size analysis and a receiver operating characteristic model, Fusobacterium was suggested as a potential biomarker for CRC screening. Additionally, we found that surgery greatly reduced the bacterial diversity of microbiota in CRC patients. Some commensal beneficial bacteria of the intestinal canal, such as Faecalibacterium and Prevotella, were decreased, whereas the drug-resistant Enterococcus was visibly increased in CRC post-surgery group. Meanwhile, we observed a declining tendency of Fusobacterium in the majority of follow-up CRC patients who were still alive approximately 3 y after surgery. We also observed that beneficial bacteria dramatically decreased in CRC patients that recidivated or died after surgery. This revealed that important bacteria might be associated with prognosis.

Conclusions: The fecal bacterial diversity was diminished in CRC patients compared with that in NC. Enrichment and depletion of several bacterial strains associated with carcinomas and inflammation were detected in CRC samples. Fusobacterium might be a potential biomarker for early screening of CRC in Chinese or Asian populations. In summary, this study indicated that fecal microbiome-based approaches could be a feasible method for detecting CRC and monitoring prognosis post-surgery.
\end{abstract}

Keywords: Colorectal cancer, 16 s rRNA gene sequencing, Fusobacterium, Faecalibacterium, Prevotella, Biomarker

\footnotetext{
* Correspondence: yemingzhi@genomics.cn

${ }^{\dagger}$ Qiulin Yao and Meifang Tang contributed equally to this work.

${ }^{1}$ Clinical laboratory of BGl Health, BGI-Shenzhen, Shenzhen 518083, China

${ }^{5}$ BGI Genomics, BGI-Shenzhen, Shenzhen 518083, China

Full list of author information is available at the end of the article
}

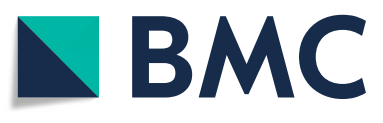

(C) The Author(s). 2021 Open Access This article is licensed under a Creative Commons Attribution 4.0 International License, which permits use, sharing, adaptation, distribution and reproduction in any medium or format, as long as you give appropriate credit to the original author(s) and the source, provide a link to the Creative Commons licence, and indicate if changes were made. The images or other third party material in this article are included in the article's Creative Commons licence, unless indicated otherwise in a credit line to the material. If material is not included in the article's Creative Commons licence and your intended use is not permitted by statutory regulation or exceeds the permitted use, you will need to obtain permission directly from the copyright holder. To view a copy of this licence, visit http://creativecommons.org/licenses/by/4.0/. The Creative Commons Public Domain Dedication waiver (http://creativecommons.org/publicdomain/zero/1.0/) applies to the data made available in this article, unless otherwise stated in a credit line to the data. 


\section{Introduction}

Colorectal cancer (CRC) is the third most common cancer worldwide, with an annual occurrence of 1,360,000 new cases and more than 600,000 deaths $[1,2]$. Because of its high incidence and mortality rate, CRC has become a major public health issue, especially in less developed regions. Moreover, survival and risk of recurrence have been reported to vary based on the stage of the tumor. According to the pathological classification, in cases of CRC confined to stages I and II, resection surgery can be curative with a 5-y survival rate of up to $80 \%$; however, the prognosis is dramatically decreased at a later stage, and the 5-y survival rate is reduced to $<10 \%$ owning to the increased occurrence of metastasis [3]. Therefore, population-wide screening and prevention programs appear to be particularly important. Fecal occult blood testing (FOBT) is currently the standard noninvasive screening test [4]. However, FOBT has certain limitations in terms of sensitivity and specificity for CRC and does not reliably detect precancerous lesions [5]. Thus, more accurate screening tests that are noninvasive and easy to perform are urgently needed for the early detection of CRC.

In recent years, owning to the widespread use of highthroughput sequencing, gut microbiota that play an important role in gut homeostasis have attracted wide attention. They have been suggested to be potentially involved in the development of CRC. Bacteria and their related products might participate in the initiation or progression of sporadic CRC by a variety of mechanisms, including induction of inflammation, production of $\mathrm{mu}$ tagenic toxins and reactive oxygen species (ROS), and the conversion of pro-carcinogenic dietary factors into carcinogens. These mechanisms have been shown to result in DNA and RNA damage, directly or indirectly inhibit DNA repair $[1,6,7]$, affect specific signal pathways, and block antitumor immunity [2]. Several bacteria have been reported to exhibit a carcinogenic risk. Escherichia coli, Streptococcus bovis, and Bacteroides fragilis are the bacteria most often described to be associated with colonic neoplasia [8, 9]. Fusobacterium nucleatum has been reported to modulate the tumor-immune microenvironment, potentiating intestinal tumorigenesis in mice [10]. In addition, some studies have indicated that F. nucleatum is enriched in the gut of CRC patients [11, 12] and have even suggested it as a putative prognostic factor in CRC [13-15].

Changes in the abundance of some gut commensal bacteria have been linked to dysbiosis observed in several human diseases. One such case regards Faecalibacterium prausnitzii, a protective bacterium, which was found to be decreased in CRC patients [8]. Culture supernatants of $F$. prausnitzii were shown to protect mice against 2,4,6-trinitrobenzenesulfonic acid-induced colitis, a potent risk factor for colon cancer [16]. All these studies suggest that $\mathrm{CRC}$ patients may have an abnormal gut microbiota structure compared with that in the normal population. Moreover, gut microbiota might be a candidate biomarker for the early detection of CRC. The test of fecal samples, in which the microorganism composition is known to be highly correlated with the colonic lumen and mucosa, seems to be an ideal approach, as these data can provide a periscopic view of gut microbial communities [17], without the need for invasive procedures, such as colonoscopies.

To date, a large amount of research has focused on the gut microbiota of CRC patients; however, the microenvironmental changes in the colorectum of patients after therapy, such as surgery, chemotherapy, or radiotherapy, have not been widely studied. As specific bacteria might drive tumorigenesis, we aimed to identify whether the population of these bacteria were decreased after effective treatment. If so, it would indicate that effective treatment might result in the alteration of the microbiota of CRC patients to one more similar to that of normal samples.

Thus, to understand the structure of the gut microbial community and the changes post-surgery in CRC patients, we investigated the microbiota in the stools of CRC patients, CRC patients after surgery, and normal controls using $16 \mathrm{~S}$ rDNA amplicon sequencing.

\section{Results}

\section{Summary of the study}

Our study population was composed of 33 normal controls (NC), 38 CRC patients before treatment (Fa), and $36 \mathrm{CRC}$ patients after surgery (Fb) (Table 1). We obtained a total of 4,992,311 $16 \mathrm{~S}$ rDNA sequences from 107 stool samples, with an average of $46,657 \pm 2955$ reads per sample in the whole cohort.

We generated 1108 total operational taxonomic units (OTUs) at a 97\% similarity level, with an average of $244 \pm 67,190 \pm 74,130 \pm 60$ OTUs in the NC, Fa, and Fb groups, respectively. The maximum number of OTUs for a single sample was 401, whereas the minimum, which was found in the Fb group, was only 27 (Table S1). There were 495 common OTUs in all groups, with the Fa group having the most specific OTUs, whereas the $\mathrm{Fb}$ group having the least specific OTUs (Figure S1).

\section{Richness and diversity}

The observed species and Chao richness index, Shannon, and Simpson diversity index were used to describe the alpha diversity features of the bacterial communities in our samples. We observed that the species richness and diversity in $\mathrm{Fa}$ and $\mathrm{Fb}$ were decreased compared with those in the NC group. A strong decrease in biodiversity was observed in the Fb group, especially in the stages II 
Table 1 Demographic structure and clinical data of the study population

\begin{tabular}{llll}
\hline & Fa & Fb & NC \\
\hline Number & 38 & 36 & 33 \\
Male, $\mathbf{n}(\%)$ & $24(63.16)$ & $23(63.89)$ & $17(51.52)$ \\
Mean age ( \pm SD, y) & $64.32 \pm 11.14$ & $63.19 \pm 10.73$ & $59.00 \pm 4.94$ \\
Pathological stage /II/III (\%) & $13.51 / 35.14 / 51.35$ & $8.82 / 44.12 / 47.06$ & - \\
\hline
\end{tabular}

Fa CRC patients before treatment, $F b$ CRC patients after surgery, NC normal controls, SD standard deviation

and III subgroup of $\mathrm{Fb}$, compared with that in the other groups (Figs. 1a, S2).

We used analysis of similarities (ANOSIM) to estimate the similarity among groups. Our results indicated that differences among groups were more significant than differences within groups (R-value $=0.164, P=0.001$ ) (Fig. 1b). At the same time, a beta diversity evaluation, represented by partial least squares discrimination analysis (PLSDA), showed a clustering of samples according to disease with an obvious separation between the $\mathrm{Fa}$ and $\mathrm{NC}$ groups, and the $\mathrm{Fb}$ and $\mathrm{NC}$ groups, but a partial separation between the $\mathrm{Fa}$ and $\mathrm{Fb}$ groups. Permutational multivariate analysis of variance confirmed this observation (ADONIS, Fa-NC, $R^{2}=0.11797, P=0.001$; Fa-Fb, $R^{2}=0.05057, P=0.001 ; \mathrm{Fb}-\mathrm{NC}, R^{2}=0.18593, P=$ 0.001) (Fig. 1c).

\section{Bacterial microbiota composition}

We analyzed the composition and abundance of bacteria at all taxonomic levels. As expected, we found that a large majority of the bacteria in the $\mathrm{Fa}, \mathrm{Fb}$, and $\mathrm{NC}$ groups belonged to the phyla Bacteroidetes, Firmicutes, Proteobacteria, Fusobacteria, and Actinobacteria. We further identified that the distribution of the major phyla in the NC group was consistent with published data. Further comparison of the relative abundance revealed

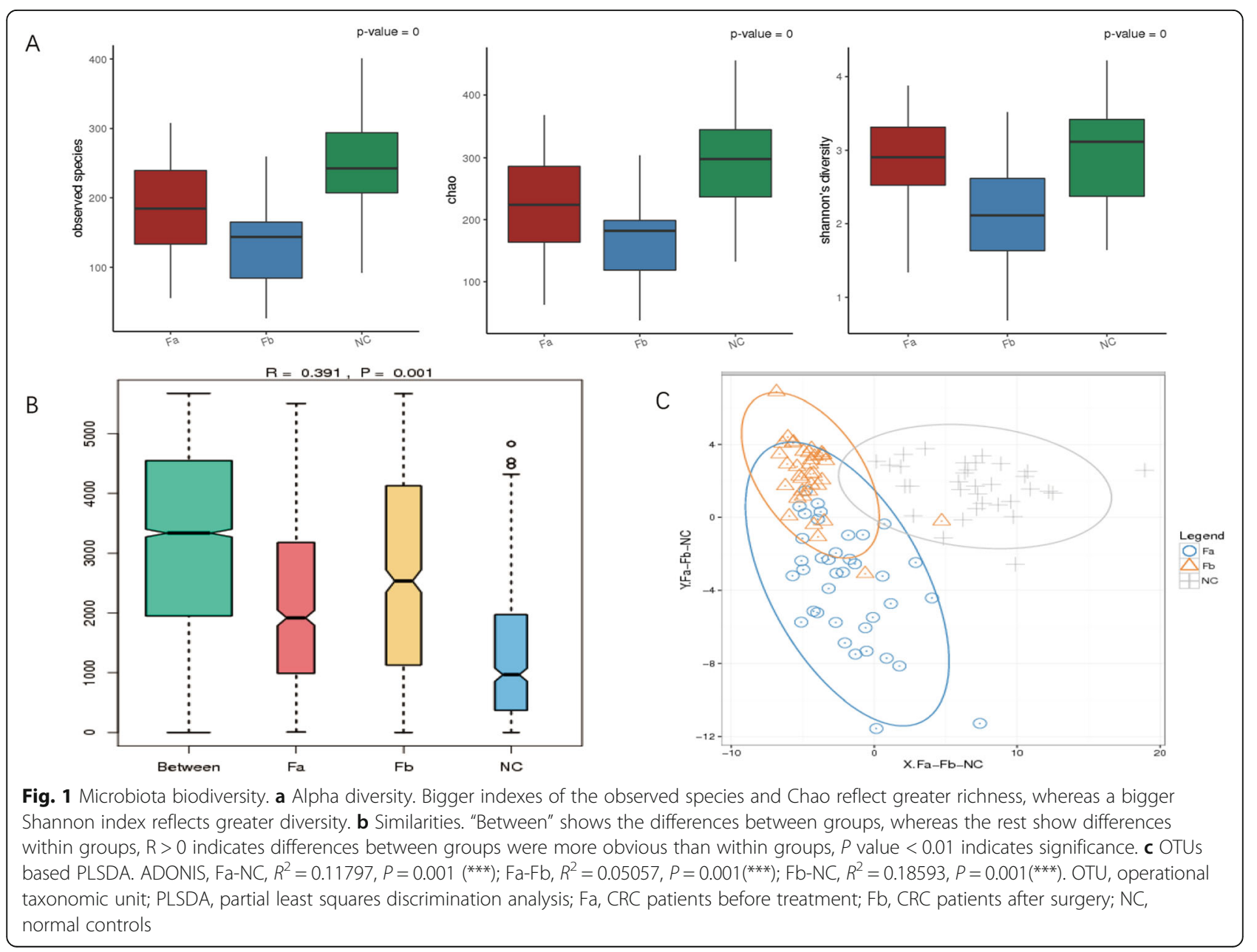


clear differences. The most abundant phylum in the NC group was Bacteroidetes, followed by Firmicutes, Proteobacteria, and Actinobacteria. However, an increase in the distribution of Proteobacteria, Fusobacteria, and Verrucomicrobia was observed in the Fa group. Except for an increase in the distribution of Fusobacteria and Verrucomicrobia, the $\mathrm{Fb}$ group was characterized by a notable increase in Proteobacteria and a decrease in Firmicutes (Figure S3).

We identified a total of 173 genera at the genus level, with the dominant genus among all groups being Bacteroides $(34.89,32.34$, and $22.89 \%$ in $\mathrm{NC}, \mathrm{Fa}$, and $\mathrm{Fb}$ groups, respectively) (Table S2). However, apart from this, the composition and prevalence of genera was different among the three groups. In the NC group, we identified the following genera: Prevotella (21.49\%), Faecalibacterium (8.58\%), Roseburia (3.28\%), and Ruminococcus $(3.02 \%)$. The Fa group was characterized by the presence of Escherichia (10.69\%), Faecalibacterium (5.49\%), Prevotella (4.78\%), and Parabacteroides (3.84\%); whereas in the $\mathrm{Fb}$ group, we observed Escherichia (18.56\%), Parabacteroides (6.81\%), Enterococcus (5.82\%), and Morganella (4.68\%) (Table S2). Accordingly, Escherichia belonging to the phylum Proteobacteria, Fusobacterium belonging to Fusobacteria, and Parabacteroides were found to be enriched in CRC patients ( $\mathrm{Fa}$ and $\mathrm{Fb}$ groups) compared with that in the NC group, whereas Prevotella was demonstrated to be overrepresented in the NC group. The presence of Faecalibacterium was scarce, whereas Enterococcus was abundant in the $\mathrm{Fb}$ group (Fig. 2). Although Bacteroides exhibited a similar relative abundance at the genus level, at the species level, the abundance of $B$. fragilis was shown to vary among groups (Figure $\mathrm{S} 4$ ), being enriched in the $\mathrm{Fa}$ and $\mathrm{Fb}$ groups.

\section{Identification of differential microbes and key taxa (biomarkers)}

To identify the key bacteria causing divergence between different groups, we used the linear discriminant analysis (LDA) effect size (LEfSe) biomarker discovery tool, which could compare two or more groups, and search for biomarkers showing statistical differences. We performed LEfSe analysis at both the family and genus levels, and found 52 discriminative features using a threshold of LDA score of $2(P$ value $<0.01)$ at the genus level (Table S3). We observed that Prevotella (LDA $=$ 4.95, $P<0.01)$, Faecalibacterium $(\mathrm{LDA}=4.66, P<0.001)$, Roseburia (LDA $=4.23, P<0.001)$, Megamonas $(\mathrm{LDA}=$ 4.21, $P<0.001)$, and Sutterella $(\mathrm{LDA}=4.08, P<0.001)$ were the dominant microbes in the NC group; Fusobacterium (LDA $=4.22, P<0.001$ ) was the dominant genus in the Fa group, whereas Escherichia (LDA $=4.96, P<$ 0.001), Parabacteroides (LDA $=4.54, P<0.001$ ), Enterococcus $(\mathrm{LDA}=4.49, P<0.001)$, Morganella $(\mathrm{LDA}=4.43$, $P<0.001)$ and Stenotrophomonas (LDA $=4.17, P<0.01$ ) were the dominant genera in the $\mathrm{Fb}$ group. The genera with an LDA score higher than 3 are displayed in Fig. 3a. To further compare the relative abundance of these primary biomarkers in all groups, we evaluated the average relative abundance of bacteria with an LDA score higher than 4 in every group. Except for Bacteroides, all other bacteria showed significant differences $(P<0.01)$ (Fig. 3b).

Consecutively, to explore whether these differential microbes were suitable for CRC detection, or

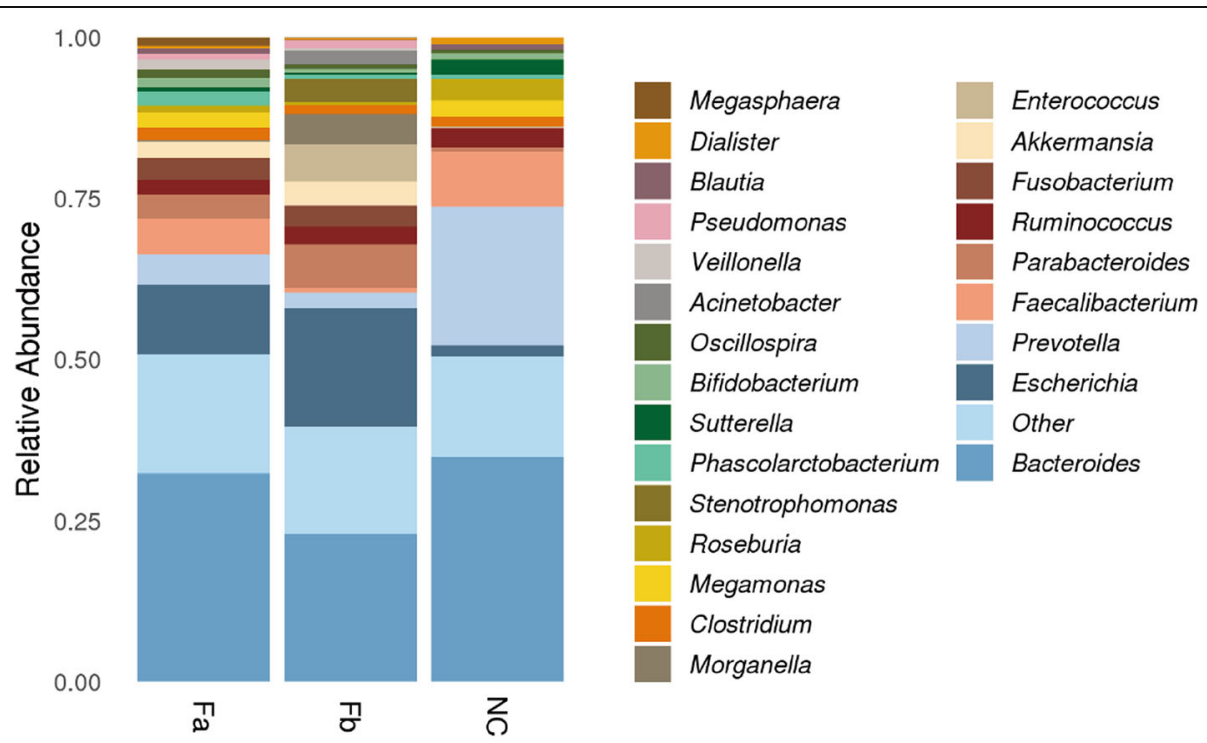

Fig. 2 Microbiota composition in every group at the genus level. Relative abundances of less than 0.5\% were combined and shown as other. Fa, CRC patients before treatment; Fb, CRC patients after surgery; NC, normal controls 


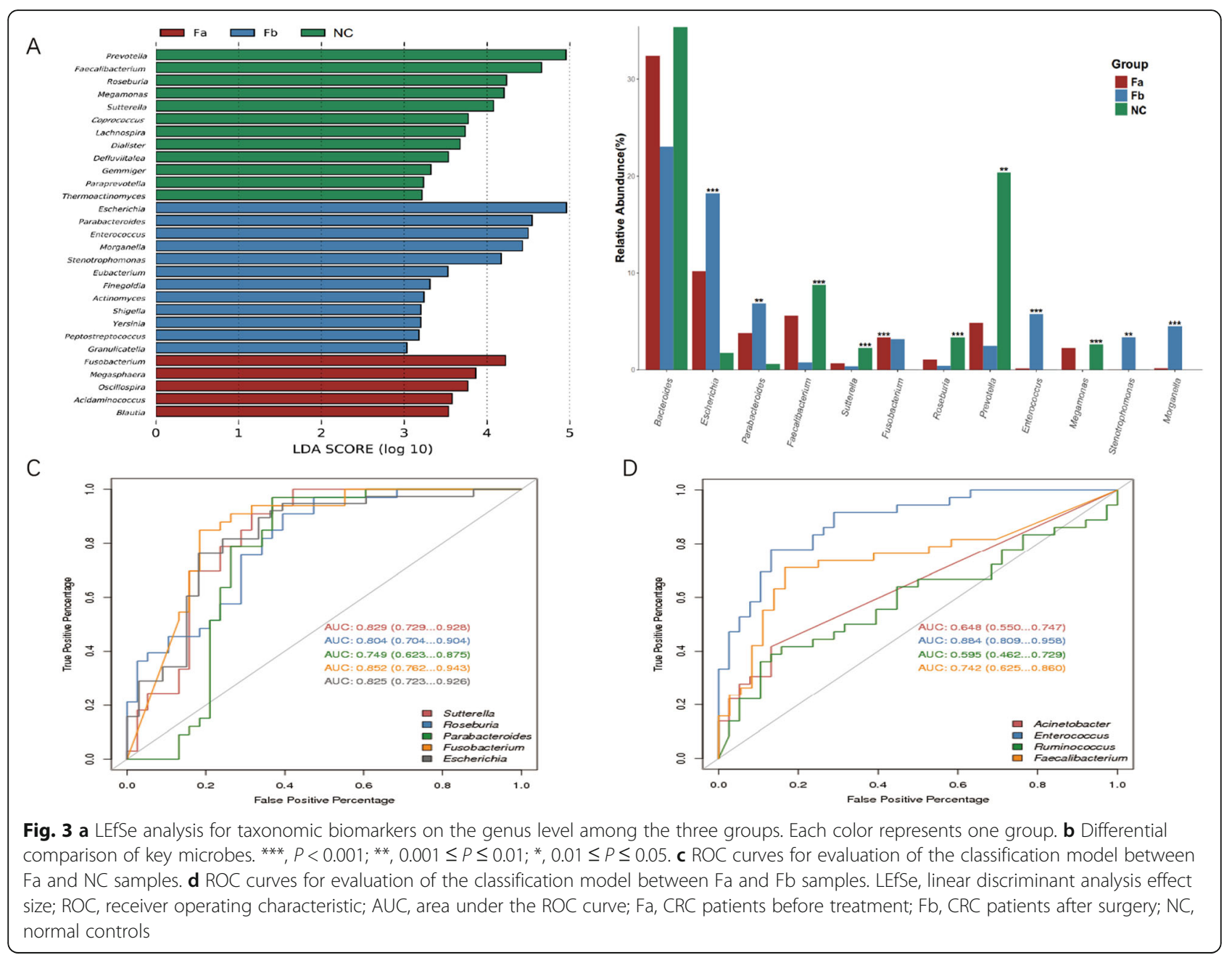

classification of CRC samples before or after treatment, we used the receiver operating characteristic (ROC) curve to evaluate their predictive power. First, we calculated the area under the ROC curve (AUC) of the microbes between the $\mathrm{Fa}$ and $\mathrm{NC}$ groups, and found that the most discriminative genus was that of Fusobacterium with an AUC of 0.852 (Fig. 3c). Consistent with previous studies suggesting Fusobacterium as prevalent in the gut of CRC patients [11, 12], potentially accelerating tumorigenesis [10], our results further confirmed this enrichment and indicated a potential biomarker for detecting CRC. Following, we evaluated the classification model comparing samples from before (Fa group) and after (Fb group) treatment. Our results revealed that the most discriminative microbe, which was shown to be enriched in the $\mathrm{Fb}$ group, with an AUC value of 0.884, was Enterococcus (Fig. 3d).

\section{Function analysis and correlation with clinic data}

We used Picrust2 to predict the MetaCyc pathways of microbiota in every sample. This analysis revealed the differential functions between CRC patients and healthy individuals and between CRC patients before and after treatment. We observed that the pathways enriched in the Fa group compared with those of the NC group were those of lipid, fatty acid, amino acid, aldehyde, alcohol, and aromatic compound degradation (Fig. 4a). Only the metabolic regulator biosynthesis as well as fatty acid and lipid degradation pathways were identified to be significantly $(P<0.01,|\log 2 \mathrm{FC}|>1)$ different in the $\mathrm{Fa}$ and $\mathrm{Fb}$ groups. (Fig. 4b).

We collected 56 clinical indexes of CRC patients, including biochemical criteria and routine blood examinations. At the genus level, 27 differentially abundant microbes (LDA score $>3, P<0.01$ ) were selected and related to the clinical data. We calculated the Pearson coefficient of pairwise correlation between microbes and clinical indexes, and characters exhibiting a high correlation (Pearson coefficient $\geq 0.7$ ) are displayed on a heatmap (Fig. 4c). A similar abundance model and very strong correlation could be observed in some microbes, such as Escherichia, Enterococcus, Yersinia, and 


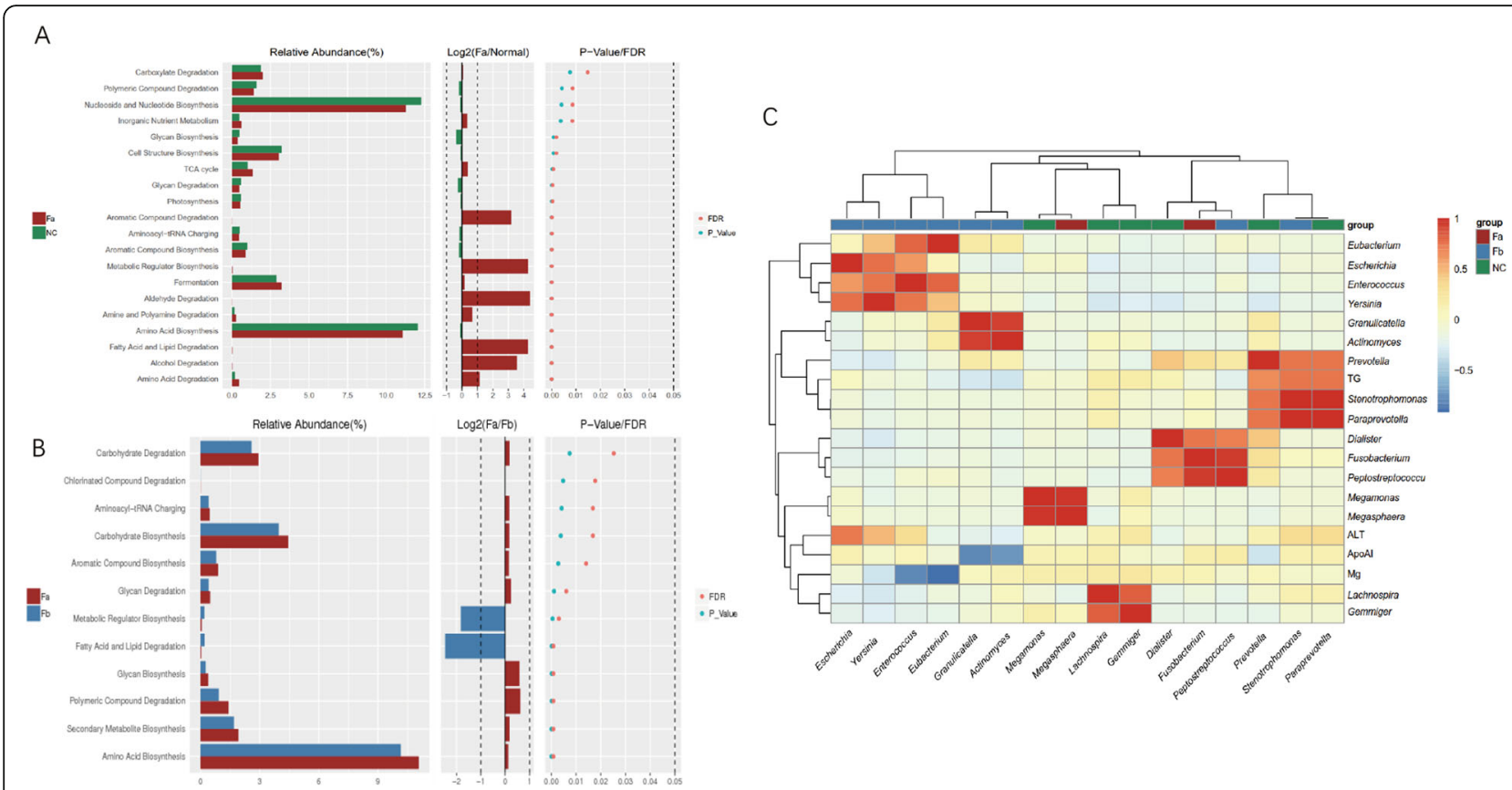

Fig. 4 a Comparison of differential functional pathways in the Fa and NC groups. $\mathbf{b}$ Comparison of differential functional pathways in the Fa and Fb groups. c Heatmap of correlation. Fa, CRC patients before treatment; Fb, CRC patients after surgery; NC, normal controls

Eubacterium, which were enriched and clustered well in the Fb group, whereas Fusobacterim and Peptostreptococcus were found to be clustered together, with a Pearson coefficient of 0.95. We selected ALT, ApoA1, $\mathrm{Mg}^{2+}$, and TG, among all the clinical indexes. ALT was shown to be positively related to Escherichia $(R=0.7303)$, TG was positively related to Stenotrophomonas $(R=0.7333)$ and Paraprevotella $(R=0.7328)$, whereas $\mathrm{Mg}^{2+}$ was negatively related to Eubacterium $(R=-0.9096)$ and Enterococcus $(R=-0.7795)$, and ApoA1 was negatively related to Granulicatella $(R=-0.7785)$ and Actinomyces $(R=-0.7336)$.

\section{Biomarkers and prognosis}

We followed-up 32 CRC patients who had provided preand post-treatment stool samples, and recorded their current living state (approximately 3 y after surgery). We evaluated the changes in important bacteria in paired stool samples. We observed a decrease in the presence of Fusobacterium in most patients treated at the SUN YAT-SEN University Cancer Center after surgery, except for two cases of distinct increases. One CRC patient died after surgery, while the other had chronic enteritis (Fig. 5a). Meanwhile, most patients treated at the SUN YAT-SEN Memorial Hospital exhibited the same decrease in Fusobacterium after surgery (Fig. 5b). Five patients (A147, A119, B103, B106, B112) either developed postoperative recidivation or died. We observed that most of the samples showed an abnormal increase in Fusobacterium, and all of them exhibited an obvious decrease in beneficial bacteria (Faecalibacterium and Prevotella) (Fig. 5).

\section{Discussion}

In this study, we compared the fecal microbiota of CRC patients to those of NC and CRC patients that underwent surgery. We observed changes in the microbiota in all three groups. The richness and biodiversity among these groups was found to differ. We detected a decrease in richness and diversity in the Fa group compared with those in the NC group. In addition, a beta diversity evaluation showed an obvious distinction between $\mathrm{Fa}$ and $\mathrm{NC}$ groups. The results indicated that gut microbiota dysbiosis occurred in CRC patients. However, because some patients were subjected to endoscopic intervention less than 1 month before sampling, we could not eliminate the possible bias due to invasive operation. Because once a patient is diagnosed, the operation is performed immediately after making an appointment with the doctor. In particular, we detected a strong decrease in biodiversity in CRC patients who had undergone surgical operation (Fb group, approximately 1 wk. after surgery), indicating that surgery might lead to serious microbiota dysbiosis and reduce biodiversity. However, as patients in the $\mathrm{Fb}$ group were administered antibiotics after surgery, we could not eliminate the effect of antibiotics on biodiversity.

We also observed obvious differences in bacterial composition. The composition of fecal microbiota in the $\mathrm{Fa}$ and $\mathrm{Fb}$ groups was clearly different from that in the $\mathrm{NC}$ 
A

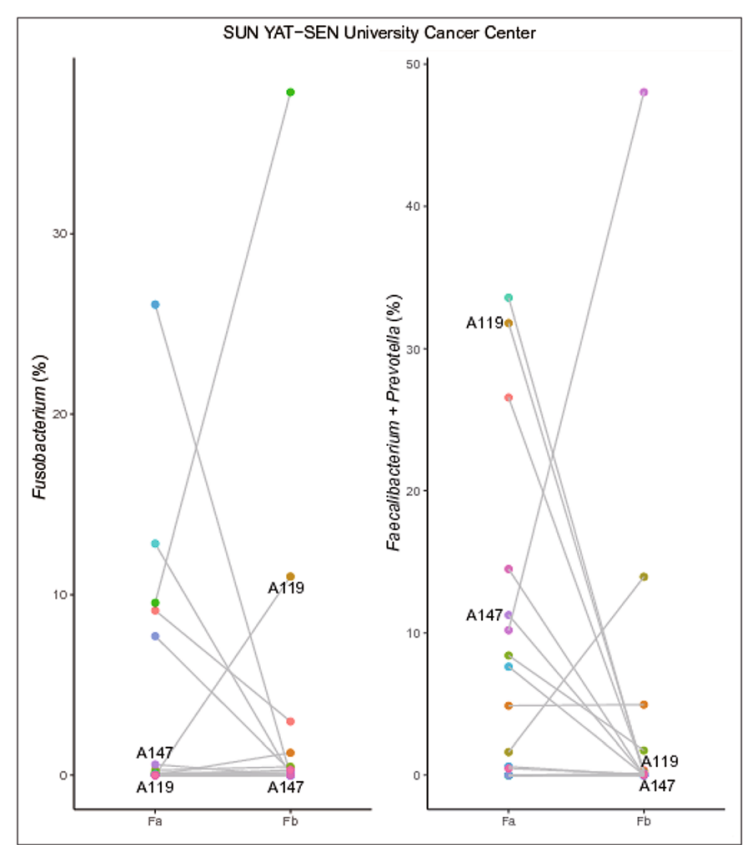

B

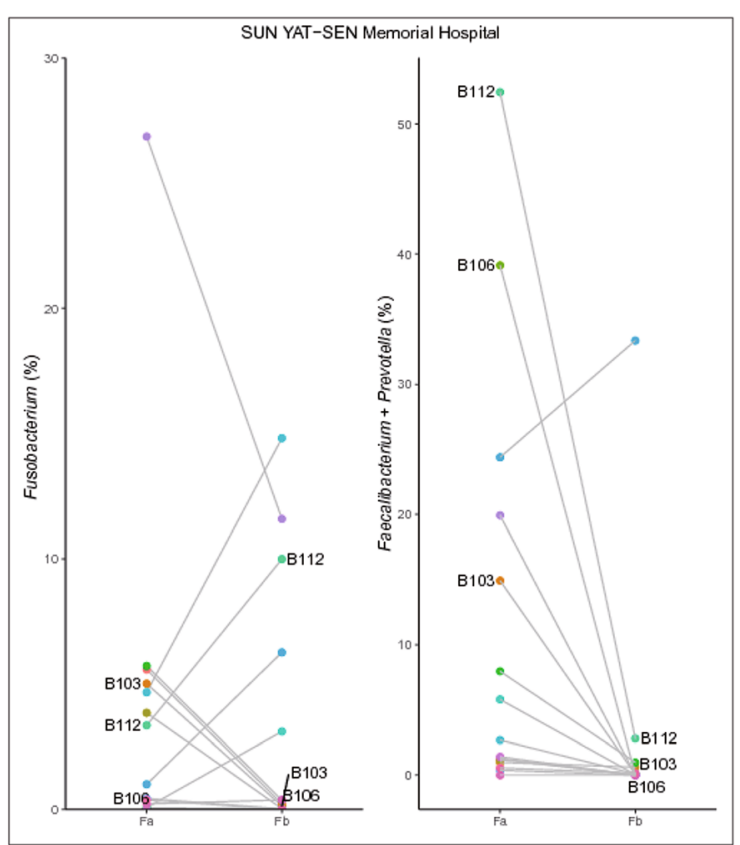

Fig. 5 Relative abundance of Fusobacterium, Faecalibacterium, and Prevotella in every paired sample before and after surgery. a Samples collected from the SUN YAT-SEN University Cancer Center. b Samples collected from the SUN YAT-SEN Memorial Hospital. Fa, CRC patients before treatment; Fb, CRC patients after surgery

group, with a clear increase in the abundance of Escherichia, Parabacteroides, and Fusobacterium being observed in both the $\mathrm{Fa}$ and $\mathrm{Fb}$ groups. Previous studies have reported a number of microbial species found in CRC patients, most of which were present in our dataset as well. For instance, Fusobacterium was reported to coexist with tumors, and was considered to positively regulate tumor cell propagation $[12,13]$. F. nucleatum was demonstrated to increase the tumor burden and selectively expand myeloid derived immune cells, such as

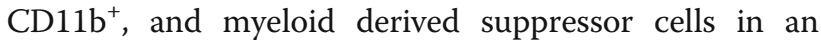
$\mathrm{Apc}^{\mathrm{Min} /+}$ mouse model [10]. Other studies suggested that through the recruitment of tumor-infiltrating immune cells, Fusobacterium might generate a proinflammatory microenvironment that is conducive for colorectal neoplasia progression. In accordance with these findings, our results showed that Fusobacterium was identified to be the principal genus in the Fa group. Moreover, the classification model between the $\mathrm{Fa}$ and NC groups was credible with an AUC of 0.852, suggesting that Fusobacterium could be used as a potential biomarker for CRC patients. Thus, the increased abundance of Fusobacterium could be linked with a high risk of CRC. Enterotoxigenic B. fragilis has been identified as a potential driver of CRC in both human and mouse studies [18-20]. The toxin of B. fragilis is known to cause human inflammatory diarrhea. However, it can also asymptomatically colonize a proportion of the human population, thereby triggering colitis and strongly inducing colonic tumors via activation of the T-helper type $17 \mathrm{~T}$-cell responses [20]. In our dataset, we found that the species of $B$. fragilis was prominent in CRC patients either before or after surgery, especially in the Fb group. E. coli is a commensal bacterium of the human gut microbiota, but some pathogenic strains have acquired the ability to induce chronic inflammation or produce toxins, such as cyclomodulins, which could participate in carcinogenesis processes $[1,21,22]$. We also tested the enrichment of Escherichia in CRC patients, especially in the $\mathrm{Fb}$ group. At the species level, the presence of $E$. coli was shown to be obviously increased in the $\mathrm{Fa}$ and $\mathrm{Fb}$ groups than in the NC group. All the aforementioned bacteria involved in CRC are known to be proinflammatory-associated, and hence might colonize faster in an inflammatory conducive environment. We also demonstrated the enrichment of these bacteria in CRC patients (Fa group) in our study. Besides, as the fecal samples of the $\mathrm{Fb}$ group in this study were obtained from CRC patients who had recently undergone surgery, their intestinal microenvironments were probably unstable, with some potentially exhibiting an inflammatory response, and some may have bad prognosis. Therefore, our findings that the abundance of $B$. fragilis and $E$. coli was the largest in the $\mathrm{Fb}$ group, whereas Fusobacterium showed a slight decrease compared with that in the Fa group, was justified. The distribution trend 
of these bacteria after surgery should be analyzed in different stages post-surgery, and thus further research is needed.

In our study, the number of Faecalibacterium and Prevotella was reduced in CRC patients, especially in the $\mathrm{Fb}$ group. We further noted that this reduction was more notable in patients who recrudesced or died after surgery. F. prausnitzii is one of the most abundant bacteria in the human intestinal microbiota of healthy individuals, and the most important butyrate-producing bacteria in the human colon [23], representing more than $5 \%$ of the total bacterial population [16]. Further, this bacterium has shown potential to function as a probiotic in the treatment of Crohn's disease [24]. Changes in the abundance of $F$. prausnitzii have been linked to dysbiosis in several human disorders. To date, this commensal bacterium has been considered as a bioindicator of human health. Prevotella, a commensal bacterial genus known to produce short chain fatty acids and that possesses potent anti-inflammatory effects, has been reported to be more commonly found in non-Westerners, who prefer a plant-rich diet $[25,26]$. Studies have confirmed that maternal carriage of Prevotella during pregnancy was associated with protection against food allergies in the offspring [26]. The comparisons of important bacteria between paired pre- and post-treatment stool samples showed an abnormal increase in Fusobacterium and an obvious decrease in Faecalibacterium and Prevotella in CRC patients who recrudesced or died after surgery. This finding suggested that an abnormal increase in Fusobacterium and a distinct reduction of probiotic bacteria indicates poor prognosis. This implies that microbial markers can be used for postoperative monitoring of CRC in combination with conventional surveillance strategies. Such a personalized surveillance strategy may allow the earlier detection of relapse. Because most of the patients were discharged from the hospital about 1 wk. after surgery, it was difficult to obtain samples after discharge. We only collected and studied one-stage fecal samples (about 1 wk. after surgery) of post-operative patients; however, follow up of samples at other stages' is required and these results should be confirmed through a larger cohort and prospective studies.

The genus Enterococcus is of great relevance to human health because of its role as a major causative agent of healthcare-associated infections; it includes resilient and versatile species able to survive under harsh conditions, most demonstrating intrinsic resistance to common antibiotics, such as virtually all cephalosporins, aminoglycosides and clindamycin [27]. As individuals in the $\mathrm{Fb}$ group had recently undergone resection, and were administered antibiotics, serious microbial dysbiosis might have occurred in their gut. As Enterococcus is known to exhibit versatility and drug-resistance, it could still adapt to the post-operation environment. Thus, the increase in the numbers of this bacteria in the $\mathrm{Fb}$ group was justified.

\section{Conclusions}

In summary, this study showed different composition of fecal microbiota among Chinese healthy individuals and CRC patients before and after surgery. The bacterial richness and diversity of feces was diminished in CRC patients. However, a clear increase in Fusobacterium, Escherichia and Parabacteroides was observed in both $\mathrm{Fa}$ and $\mathrm{Fb}$ groups. We further identified Fusobacterium as a potential biomarker for CRC screening. We also found changes in the abundance of Fusobacterium, Faecalibacterium, and Prevotella which were shown to be related to prognosis after surgery. These findings suggested the potential of fecal microbiota for CRC detection and postoperative surveillance. Although those results need to be validated through a larger, prospective trial, these insights may prove helpful in formulating public strategies to prevent and treat CRC, in combination with multiple factors of cfDNA methylation and alteration.

\section{Materials and methods \\ Samples}

The study protocol was approved by appropriate Institutional Review Boards (IRB) of the BGI (NO.BGIIRB15100-T1). All study participants provided written informed consent and dead patients also provided written informed consent from their legally authorized representative/next of kin. The process was in compliance with the Declaration of Helsinki, which included risk and benefit assessment. CRC patients were selected from Sun Yat-sen University Cancer Center and Sun Yat-sen Memorial Hospital, China. All CRC patients were diagnosed according to endoscopic and histological parameters. None of the patients had undergone any treatment, such as radiotherapy or chemotherapy, before enrollment. Exclusion criteria included other neoplasms, other tumor history, tuberculosis, infection by hepatitis $\mathrm{B}$ virus (HBV), hepatitis C virus (HCV), or human immunodeficiency virus (HIV), and the use of antibiotics 1 mo prior to hospitalization. The cancer stage was identified according to the TNM classification of malignant tumors. CRC patients were first enrolled and hospitalized; then, their fecal samples were collected before surgery (approximately $2-3 \mathrm{~d}$ before surgery). CRC patients underwent operative treatment, then administered cefminox sodium for $3 \mathrm{~d}$ in a row. Following collection of their fecal samples approximately $1 \mathrm{wk}$. after resection surgery, then they were allowed to leave the hospital. Samples collected from healthy individuals with no history of cancer, chronic enteritis, chronic constipation, bloody 
stools, chronic appendicitis, or chronic cholecystitis were used as NC.

In total, 107 stool samples were collected and divided into the following three groups: NC group including 33 samples; Fa group including 38 samples from CRC patients before surgery; and $\mathrm{Fb}$ group including 36 samples from CRC patients from CRC patients approximately 1 wk. after surgery. Basic information regarding this study population is listed in Table 1. Among them, 32-paired samples, each paired with pre- and post-treatment stool samples was obtained from the same patient. The current survival state of these 32 patients was recorded (Table S4).

Clinical data was also collected simultaneously. In total, 56 clinical indexes of CRC patients (pre- and postsurgery corresponding to 72 stool samples) were collected, including biochemical criteria and routine blood examinations.

\section{Sample preparation and genomic DNA extraction}

None of the patients were subjected to any invasive operation, such as endoscopic or clyster, at least $5 \mathrm{~d}$ before sampling. A light diet was suggested $3 \mathrm{~d}$ before sampling. About $5 \mathrm{~g}$ of fresh stool samples were collected by patients themselves immediately after defecation using stool collection devices, and then shipped on dry ice in insulated containers to a central lab, where the samples were immediately stored at $-80^{\circ} \mathrm{C}$ until further processing. The microbiota DNA was extracted as previously described [28]. Samples were treated with lysozyme, proteinase $\mathrm{K}$, and SDS, then purified with phenolchloroform-isoamylalcohol, precipitated using glycogen, sodium acetate and cold isopropanol, washed with $75 \%$ ethanol and resuspended in $1 \times$ TE buffer. DNA integrity and purification were detected by agarose gel electrophoresis $(1 \%, 150 \mathrm{~V}, 40 \mathrm{~min})$.

\section{Library construction and next generation sequencing (NGS)}

Qualified samples were used for the library preparation process. The microbiota DNA was amplified by polymerase chain reaction (PCR) with a bacterial 16S rDNA V4 region universe primer pair (515F, 5'-GTGCCAGC MGCCGCGGTAA-3' and 806R: 5'-GGACTACHVG GGTWTCTAAT-3'). PCR was performed using the following conditions: 3 min denaturation at $94{ }^{\circ} \mathrm{C} ; 25$ cycles of denaturation at $94{ }^{\circ} \mathrm{C}$ for $45 \mathrm{~s}$, annealing at $50^{\circ} \mathrm{C}$ for $60 \mathrm{~s}$, elongation at $72^{\circ} \mathrm{C}$ for $90 \mathrm{~s}$; and final extension at $72{ }^{\circ} \mathrm{C}$ for $10 \mathrm{~min}$. The PCR products were purified using AMPure XP beads (Axygen). Barcoded libraries were generated by emulsion PCR and quantitated in the following two ways: the average molecule length was determined using the Agilent 2100 bioanalyzer instrument and the library was quantified by real-time quantitative PCR (QPCR).

The qualified libraries were sequenced using the Illumina HiSeq2500 platform with the PE250 sequencing strategy (PE251 + 8+8+251).

\section{Sequence processing}

Raw sequences were assigned to each sample based on their unique barcode and primer; subsequently, the barcodes and primers were removed. At the same time, paired-end low-quality reads were filtered based on quality score, adapter contamination, and $\mathrm{N}$ base ratio.

Paired-end clean reads were merged using FLASH (fast length adjustment of short reads, v1.2.11) [29] according to the relationship of the overlap between paired-end reads. This was done when at least $15 \mathrm{bp}$ of the read overlapped the read generated from the opposite end of the same DNA fragment, the maximum allowable error ratio of an overlap region was set as 0.1 , and merged sequences were called clean tags.

Tags were assigned to OTUs using USEARCH (v7.0.1090) software [30], and tags with $\geq 97 \%$ similarity were clustered to the same OTU. It has been reported that a singleton OTU could be obtained due to sequencing errors or chimeras generated during PCR; therefore, chimeric sequences were detected and removed using UCHIME (v4.2.40) [31] according to the match of representative OTUs to the gold database (v20110519). The abundance of each OTU was quantified using usearch global algorithm by matching all clean tags to final OTUs, and normalized using a standard number corresponding to the sample with the least sequences.

Representative OTUs were annotated using the RDP classifier (v2.2) software [32] based on the homolog of the Greengene database (v201305), with the confidence threshold set to 0.8. OTUs without annotation or annotated to polluted species were removed, and the number of effective tags and information regarding OTU taxonomic synthesis were recorded in a table for the next analysis. The structure of the bacterial community of each sample was analyzed at all levels of taxonomy, with the relative abundance less than $0.5 \%$ in all samples combined with others.

\section{Statistical analysis}

Common and specific OTUs among groups were compared and displayed using VennDiagram R (v3.1.1). Analysis of similarities were performed using Bray-Curtis in the vegan package of R (v3.5.1); comparison of differences between and within the groups was available, thereby allowing testing of the availability of grouping.

Alpha diversity was applied to analyze the complexity of species diversity of a sample using many indexes, such as observed species, Chao, Ace, Shannon, and Simpson. 
All indices of our samples were calculated using Mother (v1.31.2) [33], and comparisons among groups were performed using the Kruskal test. Observed species and Chao were selected to identify community richness, whereas Shannon was used to identify community diversity. Beta diversity $[34,35]$ was used to evaluate the differences in species complexity among different samples, and was calculated on both weighted and unweighted UniFrac using QIIME (v1.80). Partial least squares discrimination analysis (PLS-DA) was built using the mixOmics library of R (v3.2.1), which was used to estimate the classification of samples and assess the variation in study groups.

We analyzed the differential abundance at the phylum, class, order, family, genus, and species levels. Differential abundance analysis was performed using LEfSe [36], with the $P$ value less than 0.01 and an LDA score more than 2 being considered significant. To quantify the effective size of the differential taxa, we used the fold change of the mean relative abundance between groups. Comparisons between probabilities, as well as overall differences in the mean relative abundance of each taxon between the two groups ( $\mathrm{Fa}-\mathrm{Fb}, \mathrm{Fa}-\mathrm{NC}$, or $\mathrm{Fb}-\mathrm{NC}$ ) were evaluated using a paired Wilcoxon rank sum test. Comparisons among three or more groups, such as comparisons among $\mathrm{Fa}, \mathrm{Fb}$ and $\mathrm{NC}$ groups were performed using the Kruskal-Wallis test.

The ROC curve was used to assess the confidence level of the classification model. Accordingly, ROC analysis and the AUC values were calculated using the pROC package of R.

MetaCyc pathway prediction was performed using Picrust2, MetaCyc (https://metacyc.org/) containing pathways involved in primary and secondary metabolism, related metabolites, and enzymatic reactions. Differential functions were analyzed using the Wilcox-test between the two groups. Correlation was tested by Pearson's coefficient using the R package.

\begin{abstract}
Abbreviations
CRC: Colorectal cancer; FOBT: Fecal occult blood testing; ROS: Reactive oxidative species; OTUs: Operational taxonomic units; LDA: Linear discriminant analysis; LEfSe: Linear discriminant analysis effect size; ROC: Receiver operating characteristic; AUC: Area under the ROC curve; NGS: Next generation sequencing; PCR: Polymerase chain reaction; QPCR: Quantitative PCR; FLASH: Fast length adjustment of short reads; PLSDA: Partial least squares discrimination analysis; ALT: Alanine transaminase; ApoA1: Apolipoprotein A1; TG: Triglyceride
\end{abstract}

\section{Supplementary Information}

The online version contains supplementary material available at https://doi. org/10.1186/s12866-021-02182-6.

Additional file $\mathbf{1}$ Table S1. OTUs stat of all groups. Table S2. Bacterial microbiota composition of each group at the genus level. Table $\mathbf{S 3}$.

Feature list of LefSe analysis. Table S4. Survival state and basic

information of 32 patients (paired samples). Figure S1. Venn of OTUs in all groups. Figure S2. Alpha diversity. Observed species, Chao and Ace reflected community richness; Shannon and Simpson reflected community diversity; Good's coverage reflected the sequencing coverage. Figure S3. Microbiota composition of each group at the phyla level. The relative abundance less that $0.5 \%$ in all samples were combined as others. Figure S4. Microbiota composition of each group at the species level. The relative abundance less that $0.5 \%$ in all samples were combined as others.

\section{Acknowledgements}

We acknowledge the volunteers who participated in our study.

\section{Authors' contributions}

Qiulin Yao: Methodology, Data curation, Formal analysis, Visualization, Writing-Original draft preparation. Meifang Tang: Conceptualization, Methodology, Supervision, Writing-Review \& Editing. Liuhong Zeng: Methodology, Selecting samples, Communication. Zhonghua Chu: Conceptualization, Resources. Hui Sheng: Resources. Yuyu Zhang: Resources. Yuan Zhou: Library preparation. Hongyun Zhang: Review. Huayan Jiang: Providing information. Mingzhi Ye: Funding acquisition, Resources. The author(s) read and approved the final manuscript.

\section{Funding}

This research was supported by the Guangzhou Science and Technology Plan Projects (Health Medical Collaborative Innovation Program of Guangzhou) (grant No. 201803040019, 201400000004-5), and Guangzhou Key Laboratory of Cancer Trans-Omics Research (GZ2012, NO348).

\section{Availability of data and materials}

The data reported in this study are also available in the CNGB Nucleotide Sequence Archive (CNSA: https://db.cngb.org/cnsa; accession number CNP0001385).

\section{Declarations}

Ethics approval and consent to participate

The study was approved by appropriate Institutional Review Boards (IRB) of the BGI (NO. BGI-IRB15100-T1).

\section{Consent for publication}

Not applicable.

\section{Competing interests}

Authors declare no conflicts of interests.

\section{Author details}

${ }^{1}$ Clinical laboratory of BGI Health, BGI-Shenzhen, Shenzhen 518083, China. ${ }^{2}$ BGI Education Center, University of Chinese Academy of Sciences, Shenzhen 518083, China. ${ }^{3}$ Guangdong Provincial Key Laboratory of Malignant Tumor Epigenetics and Gene Regulation, Department of Gastrointestinal Surgery, Sun Yat-sen Memorial Hospital, Sun Yat-sen University, Guangzhou 510060, China. ${ }^{4}$ Department of Experimental Research, State Key Laboratory of Oncology in South China, Collaborative Innovation Center for Cancer Medicine, Sun Yat-Sen University Cancer Center, Guangzhou 510060, China. ${ }^{5} \mathrm{BGl}$ Genomics, BGI-Shenzhen, Shenzhen 518083, China. ${ }^{6} \mathrm{BGI}$-Guangzhou Medical Laboratory, BGI-Shenzhen, Zone B Room 401, Qinglan Street, Panyu District, Guangzhou 510006, China.

Received: 17 November 2020 Accepted: 29 March 2021

Published online: 27 May 2021

References

1. Bonnet M, Buc E, Sauvanet P, Darcha C, Dubois D, Pereira B, et al. Colonization of the human gut by $E$. coli and colorectal cancer risk. Clin Cancer Res. 2014;20(4):859-67. https://doi.org/10.1158/1078-0432. CCR-13-1343.

2. Brennan CA, Garrett WS. Gut microbiota, inflammation, and colorectal cancer. Annu Rev Microbiol. 2016;70(1):395-411. https://doi.org/10.1146/a nnurev-micro-102215-095513. 
3. O'Connell JB, Maggard MA, Liu JH, Etzioni DA, Ko CY. Are survival rates different for young and older patients with rectal cancer? Dis Colon Rectum. 2004;47(12):2064-9. https://doi.org/10.1007/s10350-004-0738-1.

4. Levin B, Lieberman DA, McFarland B, Smith RA, Brooks D, Andrews KS, et al. Screening and surveillance for the early detection of colorectal cancer and adenomatous polyps, 2008: A joint guideline from the American Cancer Society, the US multi-society task force on colorectal Cancer, and the American College of Radiology. CA Cancer J Clin. 58(3):130-60. https://doi. org/10.3322/CA.2007.0018

5. Faivre J, Dancourt V, Lejeune C, Tazi MA, Lamour J, Gerard D, et al. Reduction in colorectal cancer mortality by fecal occult blood screening in a French controlled study. Gastroenterology. 2004;126(7):1674-80. https:// doi.org/10.1053/j.gastro.2004.02.018.

6. Collins D, Hogan AM, Winter DC. Microbial and viral pathogens in colorectal cancer. Lancet Oncol. 2010;12:504-12.

7. Arthur JC, Perez-Chanona E, Muhlbauer M, Tomkovich S, Uronis JM, Fan TJ, et al. Intestinal inflammation targets cancer-inducing activity of the microbiota. Science. 2012;338(6103):120-3. https://doi.org/10.1126/ science.1224820

8. Mathias LR, Giuseppina L, Bruno L, et al. Mucosa-associated microbiota dysbiosis in colitis associated cancer. Gut Microbes. 2018;2:131-42.

9. Cuevas-Ramos G, Petit CR, Marcq I, Boury M, Oswald E, Nougayrede J-P. Escherichia coli induces DNA damage in vivo and triggers genomic instability in mammalian cells. Proc Natl Acad Sci U S A. 2010;107(25): 11537-42. https://doi.org/10.1073/pnas.1001261107.

10. Kostic AD, Chun E, Robertson L, Glickman JN, Gallini CA, Michaud M, et al. Fusobacterium nucleatum potentiates intestinal tumorigenesis and modulates the tumor-immune microenvironment. Cell Host Microbe. 2013; 14(2):207-15. https://doi.org/10.1016/j.chom.2013.07.007.

11. Gao R, Kong C, Huang L, Li H, Qu X, Liu Z, et al. Mucosa-associated microbiota signature in colorectal cancer. Eur J Clin Microbiol Infect Dis. 2017;36(11):2073-83. https://doi.org/10.1007/s10096-017-3026-4.

12. Zackular JP, Rogers MA, Ruffin MT 4th, Schloss PD. The human gut microbiome as a screening tool for colorectal cancer. Cancer Prev Res. 2014; 7(11):1112-21. https://doi.org/10.1158/1940-6207.CAPR-14-0129.

13. Mima K, Cao Y, Chan AT, Qian ZR, Nowak JA, Masugi Y, et al. Fusobacterium nucleatum in colorectal carcinoma tissue according to tumor location. Clin Transl Gastroenterol. 2016;7(11):e200. https://doi.org/10.1038/ctg.2016.53.

14. Zeller G, Tap J, Voigt AY, Sunagawa S, Kultima JR, Costea PI, et al. Potential of fecal microbiota for early-stage detection of colorectal cancer. Mol Syst Biol. 2014;10:766. https://doi.org/10.15252/msb.20145645.

15. Mima K, Nishihara R, Qian ZR, Cao Y, Sukawa Y, Nowak JA, et al. Fusobacterium nucleatum in colorectal carcinoma tissue and patient prognosis. Gut. 2016;65(12):1973-80. https://doi.org/10.1136/gutjnl-2015-31 0101.

16. Miquel S, Martin R, Rossi O, Bermudez-Humaran LG, Chatel JM, Sokol H, et al. Faecalibacterium prausnitzii and human intestinal health. Curr Opin Microbiol. 2013;16(3):255-61. https://doi.org/10.1016/j.mib.2013.06.003. PMID: 23831042.

17. Yasuda K, Oh K, Ren B, Tickle TL, Franzosa EA, Wachtman LM, et al. Biogeography of the intestinal mucosal and lumenal microbiome in the rhesus macaque. Cell Host Microbe. 2015;17(3):385-91. https://doi.org/10.1 016/j.chom.2015.01.015.

18. Geis AL, Fan H, Wu X, Wu S, Huso DL, Wolfe $J$, et al. Regulatory T-cell response to enterotoxigenic Bacteroides fragilis colonization triggers IL17dependent colon carcinogenesis. Cancer Discov. 2015;5(10):1098-109. https://doi.org/10.1158/2159-8290.CD-15-0447.

19. Goodwin AC, Destefano Shields CE, Wu S, Huso DL, Wu X, Murray-Stewart TR, et al. Polyamine catabolism contributes to enterotoxigenic Bacteroides fragilis-induced colon tumorigenesis. Proc Natl Acad Sci U S A. 2011;108(37): 15354-9. https://doi.org/10.1073/pnas.1010203108.

20. Wu S, Rhee K-J, Albesiano E, Rabizadeh S, Wu X, Yen HR, et al. A human colonic commensal promotes colon tumorigenesis via activation of $T$ helper type 17 T cell responses. Nat Med. 2009;15(9):1016-22. https://doi.org/10.1 038/nm.2015.

21. Swidsinski A, Khilkin M, Kerjaschki D, Schreiber S, Ortner M, Weber J, et al. Association between intraepithelial Escherichia coli and colorectal cancer. Gastroenterology. 1998;115(2):281-6. https://doi.org/10.1016/S00165085(98)70194-5.

22. Martin HM, Campbell BJ, Hart CA, Mpofu C, Nayar M, Singh R, et al. Enhanced Escherichia coli adherence and invasion in Crohn's disease and colon cancer. Gastroenterology. 2004;127(1):80-93. https://doi.org/10.1053/j. gastro.2004.03.054.

23. Ferreira-Halder CV, Faria AVS, Andrade SS. Action and function of Faecalibacterium prausnitzii in health and disease. Best Pract Res Clin Gastroenterol. 2017;31(6):643-8. https://doi.org/10.1016/j.bpg.2017.09.011.

24. Sokol H, Pigneur B, Watterlot $L$, Lakhdari O, Bermúdez-Humarán LG, Gratadoux JJ, et al. Faecalibacterium prausnitzii is an antiinflammatory commensal bacterium identified by gut microbiota analysis of Crohn disease patients. Proc Natl Acad Sci U S A. 2008;105(43):16731-6. https://doi. org/10.1073/pnas.0804812105.

25. Martínez I, Stegen JC, Maldonado-Gómez MX, Eren AM, Siba PM, Greenhill $A R$, et al. The gut microbiota of rural Papua new Guineans: composition, diversity patterns, and ecological processes. Cell Rep. 2015;11(4):527-38. https://doi.org/10.1016/j.celrep.2015.03.049.

26. Vuillermin PJ, O'Hely M, Collier F, Allen KJ, Tang MLK, Harrison LC, et al. Maternal carriage of Prevotella during pregnancy associates with protection against food allergy in the offspring. Nat Commun. 2020;11(1):1452. https:// doi.org/10.1038/s41467-020-14552-1.

27. Garcia-Solache M, Rice LB. The Enterococcus: a model of adaptability to its environment. Clin Microbiol Rev. 2019;32:e00058-18. https://doi.org/10.112 8/CMR.00058-18.

28. Qin J, Li Y, Cai Z, Li S, Zhu J, Zhang F, et al. A metagenome-wide association study of gut microbiota in type 2 diabetes. Nature. 2012; 490(7418):55-60. https://doi.org/10.1038/nature11450.

29. Magoc T, Salzberg S. FLASH: fast length adjustment of short reads to improve genome assemblies. Bioinformatics. 2011;27(21):2957-63. https:// doi.org/10.1093/bioinformatics/btr507.

30. Edgar RC. Search and clustering orders of magnitude faster than BLAST. Bioinformatics. 2010;26(19):2460-1. https://doi.org/10.1093/bioinformatics/ btq461.

31. Edgar RC, Haas BJ, Clemente JC, Quince C, Knight R. UCHIME improves sensitivity and speed of chimera detection. Bioinformatics. 2011;27(16): 2194-200. https://doi.org/10.1093/bioinformatics/btr381.

32. Wang Q, Garrity GM, Tiedje JM, Cole JR. Naive Bayesian classifier for rapid assignment of rRNA sequences into the new bacterial taxonomy. Appl Environ Microbiol. 2007;73(16):5261-7. https://doi.org/10.1128/AEM.0006207.

33. Patrick DS, Sarah LW, Ryabin T, Hall JR, Hartmann M, Hollister EB, et al. Introducing mothur: open-source, platform-independent, communitysupported software for describing and comparing microbial communities. Appl Environ Microbiol. 2009;75:7537-41.

34. Lozupone C, Lladser ME, Knights D, Stombaugh J, Knight R. UniFrac: an effective distance metric for microbial community comparison. ISME J. 2011; 5(2):169-72. https://doi.org/10.1038/ismej.2010.133.

35. Lozupone C, Hamady M, Kelley ST, Knight R. Quantitative and qualitative $\beta$ diversity measures lead to different insights into factors that structure microbial communities. Appl Environ Microbiol. 2007;73(5):1576-85. https:// doi.org/10.1128/AEM.01996-06.

36. Segata N, Izard J, Waldron L, Gevers D, Miropolsky L, Garrett WS, et al. Metagenomic biomarker discovery and explanation. Genome Biol. 2011; 12(6):R60. https://doi.org/10.1186/gb-2011-12-6-r60.

\section{Publisher's Note}

Springer Nature remains neutral with regard to jurisdictional claims in published maps and institutional affiliations.

\section{Ready to submit your research? Choose BMC and benefit from:}

- fast, convenient online submission

- thorough peer review by experienced researchers in your field

- rapid publication on acceptance

- support for research data, including large and complex data types

- gold Open Access which fosters wider collaboration and increased citations

- maximum visibility for your research: over $100 \mathrm{M}$ website views per year

At BMC, research is always in progress.

Learn more biomedcentral.com/submissions 